\title{
Comparison of cardiopulmonary resuscitation techniques using video camera recordings
}

\author{
C J Mann, J Heyworth
}

\begin{abstract}
Objective-To use video recordings to compare the performance of resuscitation teams in relation to their previous training in cardiac resuscitation.

Methods-Over a 10 month period all cardiopulmonary resuscitations carried out in an accident and emergency (A\&E) resuscitation room were videotaped. The following variables were monitored: (1) time to perform three defibrillatory shocks; (2) time to give intravenous adrenaline (centrally or peripherally); (3) the numbers and grade of medical and nursing staff involved in the resuscitation; (4) the experience and training of these personnel.

Results-Of 101 resuscitations recorded, 69 were carried out by the $A \& E$ team alone and 32 by the hospital cardiac arrest team. Resuscitation procedures were carried out significantly more rapidly by the former. Skills and protocols were most effectively used when the resuscitation team was led by an experienced doctor who had received specific training in cardiopulmonary resuscitation, that is, Advanced Life Support course (ALS) or Advanced Cardiac Life Support course (ACLS). Such an individual was always present at A\&E team resuscitations but in only $6 \%$ of cardiac arrest team resuscitations.

Conclusions-ALS course completion should be regarded as a vital part of the training of any doctor involved in cardiopulmonary resuscitation.

(f Accid Emerg Med 1996;13:198-199)
\end{abstract}

Key terms: cardiopulmonary resuscitation; Advanced Life Support course; Advanced Cardiac Life Support course; performance monitoring

The development of cardiopulmonary resuscitation techniques and nationally recognised resuscitation protocols is leading towards a standardised approach to cardiopulmonary resuscitation using validated techniques. ${ }^{1}$ The use of video recording techniques for the evaluation and training of staff in resuscitation has been described. ${ }^{2}$ More recently the analysis of video tapes has been used to derive time and motion information from trauma resuscitations. ${ }^{3}$ Video enables accurate analysis of resuscitations and provides assessment of the correct use of protocols and advanced resuscitation techniques. The video recording is contemporaneous and independent of the clinician involved in the resuscitation. It is therefore not influenced by personal recollection or bias and is superior to retrospective completion of the clinical record.

Recent studies ${ }^{4}$ have highlighted several important deficiencies in the management of cardiac arrest victims by junior doctors, including unfamiliarity with Resuscitation Council algorithms and recommended drug use.

In the hospital in which this study was done, victims of out of hospital cardiac arrest are resuscitated by one of two cardiac arrest teams. Between the hours of 09.00 and 22.00 resuscitations are led by senior A\&E staff (consultant, senior registrar, registrar, or staff grade ), four $(80 \%)$ of whom had successfully completed an Advanced Cardiac Life Support (ACLS) course at the time of this study. At other times the hospital cardiac arrest team is called. This team comprises the medical registrar, senior house officer (SHO), and house officer, and the intensive care unit SHO. These doctors receive three hours of training in advanced life support (ALS) protocols as part of their induction courses.

Both resuscitation teams are notified by telephone of the imminent arrival of the patient in the $A \& E$ department and are assisted in the resuscitation by the $A \& E$ nursing staff.

Using video recordings of the resuscitations we compared the performance of these two teams with specific regard to their previous training in cardiac resuscitation. The video camera used to record the resuscitations has been in the department for two years. It is sited 2 metres beyond the resuscitation trolley and gives a clear view over the whole resuscitation area. It is capable of recording speech. The camera is activated immediately before the patient's arrival by either the medical or nursing staff.

\section{Methods}

Over a 10 month period all cardiopulmonary resuscitations carried out in the resuscitation room were videotaped. These tapes were confidentially reviewed by the authors and then erased within $6 \mathrm{~d}$ in accordance with the Data Protection Act.

The following variables were monitored: (1) time to perform three defibrillatory shocks; (2) time to give intravenous adrenaline (centrally or peripherally); (3) the numbers and grade of medical and nursing staff involved in the resuscitation; and (4) the experience and training of these personnel.

\section{Results}

One hundred and one resuscitations were evaluated. Of these, 69 were carried out by the 
Personnel and interventions by team

\begin{tabular}{llcl}
\hline & AEE team & Hospital team & P value \\
\hline Time for three dc shocks & 54 seconds & 83 seconds & 0.01 \\
Time for iv adrenaline & 65 seconds & 115 seconds & 0.01 \\
Central venous access & $62(90 \%)$ & $8(25 \%)$ & $0 \cdot 01$ \\
Doctors & $2 \cdot 6$ & 3.2 & 0.05 \\
Nurses & 4 & $2 \cdot 7$ & 0.05 \\
ALS trained doctors & 1 & 0.06 & 0.01 \\
ALS trained nurses & 1.5 & 1 & NS \\
\hline
\end{tabular}

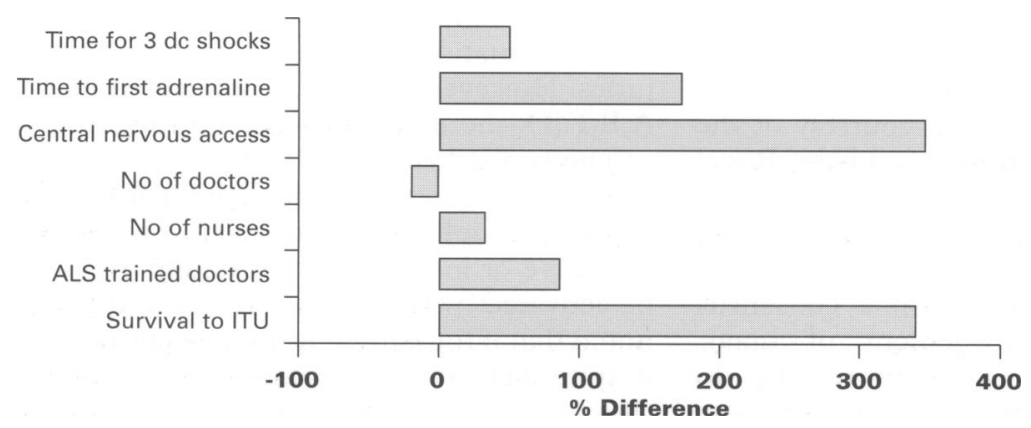

Comparison of hospital and $A \mathcal{E} E$ cardiac arrest teams.

A\&E team alone and 32 by the hospital cardiac arrest team. The table gives comparative data for the two teams.

In all cases, cardiopulmonary resuscitation, as defined by external cardiac massage and ventilation either by bag/valve/mask device or by endotracheal intubation with high flow oxygen, was established within $60 \mathrm{~s}$ of patient arrival.

There was a statistically significant difference between the two teams in the time required to deliver three dc shocks $(P<0.001)$.

The first dose of adrenaline was given through a peripheral cannula within $60 \mathrm{~s}$ of arrival in $75 \%$ (24) of patients in the hospital arrest team group and $90 \%$ (62) of patients in the $A \& E$ team group (NS). All patients received the first dose of adrenaline by peripheral route as a peripheral vein had been cannulated by the paramedics in all cases. Central venous cannulation was more frequent in the $A \& E$ team resuscitations $(P=0.01)$.

The composition of the resuscitation teams was significantly different $(P=<0 \cdot 01)$. $A \& E$ team resuscitations were always led by a doctor who had successfully completed a three day ACLS course. The doctors were supported by a mean of four nurses. The cardiac arrest teams were led by fully ALS trained staff in only $6 \%$ of cases They were attended by a mean number of $2 \cdot 7$ nurses.

Fourteen patients $(20 \%)$ left the resuscitation room with spontaneous circulation in the $A \& E$ team group compared with four patients $(12 \%)$ in the hospital team arrest group (NS).

Two patients survived to hospital discharge in the $\mathrm{A} \& \mathrm{E}$ resuscitation group. There were no discharges from hospital in the cardiac arrest team group (NS).

The performance of the two teams is compared in the figure.

\section{Discussion}

The benefit of videotape analysis is to enable objective analysis of individual and team performance, including assessment of clinical skills during resuscitation. Video analysis can also be used to validate both the content and the effectiveness of resuscitation training.

Our study has shown that there were significant differences in the performance of the two arrest teams. Importantly the increased time for defibrillation and reduced use of central venous drug administration by the hospital team is of concern since both these have been shown to affect survival. ${ }^{5}$

At the time of this study only one member of the junior medical staff had attended an ALS course, although all had received three hours of cardiopulmonary resuscitation training based on the United Kingdom Resuscitation Council guidelines.

The ALS course provides a total of 20 hours of didactic teaching, with individual tuition and rigorous assessment of both theoretical knowledge and practical skills.

Training in advanced cardiac life support skills by completion of an ALS course or equivalent improves clinical performance. The three hours of resuscitation training provided for members of the cardiac arrest team are not sufficient to match the level of proficiency achieved on an ALS course.

The effect of this approach is improved performance in the resuscitation of cardiac arrest victims. ALS course completion should therefore be regarded as a vital part of the training of any doctor involved in cardiac arrest resuscitations, especially those who will take the role of team leader.

1 Resuscitation Council (UK). ALS Course guidelines, 1991.

2 Komelasky AL, Bond BS. The effect of two forms of learning reinforcement upon retention of CPR skills. Paediatr Nurs 1993;19:96-8.

3 Mann FA, Walkup RK, Berryman CR, Bessey PQ, Wilson AJ, Vannier MW. Computer-based videotape analysis of trauma resuscitations for quality assurance and clinical trauma resuscitations for quality as
research. $\mathcal{T}$ Trauma $1994 ; 36: 226-30$.

4 research. F Trauma 1994;36:226-30. candidates. BMF 1993;306:1578-9.

5 American Heart Association. Guidelines for cardiopulmonary resuscitation (CPR) and emergency cardiac care (ECC). $\mathfrak{F} A M A$ 1992;268:2172. 